\title{
Morphological memory of polymeric bodies
}

\author{
I. V. Melikhov ${ }^{1}$, O. V. Alekseeva ${ }^{2}$, V. N. Rudin ${ }^{1}$, E. D. Kozlovskaya ${ }^{1}$, A. V. Noskov ${ }^{2}$ \\ ${ }^{1}$ M. V. Lomonosov Moscow State University, Moscow, Russia \\ ${ }^{2}$ G. A. Krestov Institute of Solution Chemistry RAS, Ivanovo, Russia \\ melikhov@radio.chem.msu.ru,ova@isc-ras.ru
}

\section{PACS 81.05.Lg}

DOI 10.17586/2220-8054-2015-6-3-412-423

The model of microrelief formation on the surface of polymers was formulated, describing the connection between the microrelief structural elements' distribution function on the states with the kinetics of macromolecule formation, aggregation and aggregates' integration into the polymer body. Methods for calculating the kinetics of these processes, using experimental data on the microrelief properties, were developed. The developed methods have proven effective in the study of microrelief on films obtained by the evaporation of $o-x y l e n e$ and toluene solutions of polystyrene, as well as polystyrene granules' microrelief. The hierarchical structures were found on the surface of these bodies, from which it was possible to "extract" information about the polymerization and aggregation of the polystyrene macromolecules. The obtained data are summarized in the form of a morphological memory representation of the polymer bodies, consisting in the long-term preservation of nonequilibrium structures available for study without destroying the body, as well as the possibility to use the results of the study to describe the kinetics of these structures' formation.

Keywords: polystyrene, microrelief, nanoparticles, subnanoparticles.

Received: 9 April 2015

\section{Introduction}

According to published results, electron and atomic force microscopy have been used to study polymeric bodies, many of which have surfaces with microrelief on their macromolecular structures. In [1], a microrelief on the surface of a film, formed by the evaporation of an $o$-xylene solution of polystyrene, was described. The microrelief of the film depended on the composition of the evaporating solution, and from this microrelief, the composition of the solution could be determined. In [2], this fact was interpreted as an indication that the film possessed a morphological memory that conferred the ability to gain the information about the conditions of its formation and the ability to use this information with the help of the data in the morphology of the microrelief. Such an interpretation requires detailed information about what was happening in the monomeric solution when the polymerization initiator was introduced. It is known that in such a solution, the nucleation and growth of the polymeric macromolecules occurs, which form the aggregates followed by their assembly into a polymeric body, and the macromolecules on the body's surface form the microrelief [3-6]. During the transition to the body structure, the condition of each macromolecule can change significantly, depending upon the relaxation time of its state $[7,8]$. As a result, if the body is formed quickly and the complete relaxation of the macromolecule state is not provided, the structure of the microrelief appears dependent on the kinetics of the body formation. All of this should be taken into account when considering the processes that lead to morphological memory. 


\section{Model of the body microrelief formation}

In this model, body formation starts when the polymerization initiator is introduced into the monomeric solution $(t=0)$ and ends at the moment when the solvent is completely removed from the system by filtration or evaporation $\left(t=t_{F}\right)$. In the range of $t=0 \div t_{F}$ the solvent, the dissolved monomers and macromolecules $(\mathrm{j}=1)$, macromolecular clusters $(\mathrm{j}=2)$, and aggregates of clusters $(\mathrm{j}=3)$ which comprise the system are uniformly distributed over the volume of the system. The state of each of these particles is characterized by parameters $\vec{X}=\{v, n, \vec{P}\}$, where $v$ is the volume, $n$ is the number of monomers included in the particle macromolecules; $\vec{P}=\left\{P_{1} \ldots P_{i} \ldots P_{m}\right\} ; P_{i}$ is one of the characteristics of the shape, composition and structure of the particle.

The states of the system are characterized by the parameters $\vec{y}=\left\{V, C_{A}, C_{B}, N_{1}, N_{2}\right.$, $\left.N_{3}, W_{V}, T\right\}$, where $V$ is its volume, $C_{A}$ and $C_{B}$ are the concentrations of the monomer and initiator molecules in the solution; $N_{1}, N_{2}, N_{3}$ are the quantities of the macromolecules, their clusters and aggregates in the system; $W_{V}$ is the rate of the system volume change; $\mathrm{T}$ is the temperature. Wherein $N_{j}=\sum_{v} N_{j}(v, t)$, if the number of $\mathrm{j}^{\text {th }}$ type particles with volume $v$ is equal to $N_{j}(v, t)$. Alternatively, in the continuum approximation, $N_{j}=\int_{v} \phi_{j}(v, t) d v$ if the particles distribution function on volume is $\phi_{j}(v, t)$.

Distribution function $\phi_{j}(v, t)$ is influenced by the events that can both increase and decrease the volume of each particle [9-12]. Therefore:

$$
\frac{\partial \phi_{j}(v, t)}{\partial t}=\Omega_{j v}-\beta_{j v} \phi_{j}(v, t)
$$

where $\Omega_{j v}$ is the frequency function characterizing the frequency of events leading to the appearance of the particles of $\mathrm{j}^{\text {th }}$ type of size $v$ in the system, that is, the frequency of particle transition in the state $(j, v) ; \beta_{j v}$ is the probability of the exit from the state $(j, v)$ per unit time.

The solution of the equation (1) with appropriate boundary conditions establishes a relationship between the frequency functions $\Omega_{j v}, \beta_{j v}$, and the total volume $V_{j}$ of the particles of each $\mathrm{j}^{\text {th }}$ type:

$$
V_{j}=\int_{v_{n j}} v \phi_{j}(v, t) d v
$$

where $v_{n j}$ is the minimum volume of the particle, to which the mentioned frequency functions (volume of the $\mathrm{j}^{\text {th }}$ type nucleus) can be ascribed.

Additionally, the condition of the conservation of the number of atoms introduced into the system is fulfilled:

$$
C_{A 0} V_{0}=\left(V-\sum_{j} V_{j}\right) C_{A}+\sum_{j} v_{0 j}^{-1} V_{j},
$$

where $C_{A 0}$ and $V_{0}$ are the initial monomer concentration and the volume of the system; $v_{0 j}$ is the average volume per one monomer in the amount of the $\mathrm{j}^{\text {th }}$ type particles.

The volume of the system, decreased at rate $W_{v}$, due to solvent removal, is equal to $V=V_{0}(1-\Phi)$, so that the condition (3) can be written in the form:

$$
C_{A 0}=\left(1-\sum_{j} \varepsilon_{j}-\Phi\right) C_{A}+\sum_{j} v_{0 j}^{-1} \varepsilon_{j},
$$


where $\varepsilon_{j}=V_{j} / V_{0} ; \Phi=\int_{0}^{t}\left(W_{v} / V_{0}\right) d t$.

According to the equations (3) and (4), at $t=t_{F}$, when $C_{A}=0$ and $\Phi=$ $\int_{0}^{t_{F}}\left(W_{v} / V_{0}\right) d t=\frac{\bar{W}_{v}}{V_{0}} t_{F}$, we have:

$$
V_{0}=\left(1-P_{F}-v_{0 S} C_{A 0}\right)=\bar{W}_{v} t_{F},
$$

where $P_{F}=V_{P} / V_{0}$ is the parameters of the body porosity at the volume of the space between the particles (pores) equal to $V_{P} ; v_{0 S}$ is the average volume per one monomer built into the body of macromolecule; $\bar{W}_{v}$ is the average speed $W_{v}$ in the interval $\left(0|t| t_{F}\right)$ dependent on $\vec{y}$.

Equation (5) defines the parameters $\vec{y}$ of the system state, at which the time $t_{F}$ remains within the range:

$$
\left(\beta_{1 v}^{-1}\left|t_{F}\right| \beta_{3 v}^{-1}\right)
$$

where $\beta_{j v}^{-1}$ is the value that characterizes the time, suitable for the particle escape from the state $(j, v)$.

Relations (1)-(5) consider the possibility of implementing multiple routes of body formation, dependent on $\vec{y}$. The simplest of these is the route in which $\beta_{1 v} \gg \beta_{2 v}, \beta_{3 v}$, $t_{F} \sim 1 / \beta_{1 v}$, and the process comes down to the formation of the polymer molecules with the volume $\varepsilon_{1}=\varepsilon_{1}(t)$ increasing up to $\varepsilon_{1}\left(t_{F}\right)=C_{A 0} v_{01}$ at the time of their association in the body of randomly arranged macromolecules. In describing the simplest route, detailing the functions $\Omega_{j v}$ and $\beta_{j v}$ applied to the chained macromolecules as described in [13,14], the equation (1) can be reduced to the form:

$$
\frac{\partial \phi_{1}(v, t)}{\partial t}=\frac{\partial^{2}\left(D_{1 v} \phi_{1 v}\right)}{\partial v^{2}}-\frac{\partial}{\partial v}\left(G_{1 v} \phi_{1 v}\right)
$$

where $D_{1 v}=\frac{1}{2}\left(\alpha_{1 v} C_{A} a_{v}^{2}+\beta_{1 v} b_{v}^{2}\right), G_{1 v}=\alpha_{1 v} C_{A} a_{v}-\beta_{1 v} b_{v}, \phi_{j v}=\phi_{j}(v, t), \alpha_{1 v}$ and $\beta_{1 v}$ are frequency functions, characterizing the frequency of events leading to an increase and decrease in volume of the particle in the state $(j=1, v)$; and are the increase and decrease of $v$ at a single event.

Due to the uniformity of the system, relations (1)-(7) characterize any of its areas, including the near-surface areas, where the microrelief of the body is formed. The parameters for the states of the surface and internal parts of the system may vary [15], but they develop according to the same rules. Therefore, we can assume that the above model describes the kinetics of microrelief formation on a mesokinetical level of detailing, that involves consideration of the distribution functions of the structural elements' microrelief on their volume.

\section{Model of the microrelief change with body usage}

When using the body its microrelief changes as a result of inevitable chemical, radiation and mechanical influence by the environment in which the body is moved. These influences can be imagined as a set of events, each of which leading to a specific increase or decrease in the volume of the particles of the near-surface areas of the body. As a result of thermal and chemical influences (e.g. collisions of the body with the molecules of the medium) or mechanical influences (e.g. contact with the bodies in the medium), the distribution function of the near-surface area particles is altered in accordance with equation (1). This equation, with reference to the microrelief, can be provided in the form:

$$
-\frac{\partial \phi_{j}(v, \tau)}{\partial \tau}=\frac{\partial}{\partial v}\left(a_{j} \omega_{j} \phi_{j v}\right)+\nu_{j} \phi_{j v}
$$


where $\phi_{j}(v, \tau)=\phi_{j v}$ is the distribution function of the particles in the near-surface area in the state $(j, v)$ at the time $\tau>t_{F}$ for the usage of the body; $\omega_{j}$ is the frequency of events leading to the change in volume $v ; a_{j}$ is the average volume change at a single event; $\nu_{j}$ is the frequency of acts of escape of $\mathrm{j}^{\text {th }}$ type particles from the near-surface areas to the medium.

Also, if the transfer of the body from the medium where it was obtained to the medium of usage takes place without violating its integrity, it is reasonable to put the solution for equation (8) in the form:

$$
\varphi_{j}(v, \tau)=\varphi_{j}\left(v, t_{F}\right) \exp \left(-\frac{\nu_{j} \tau}{\omega_{j} \tau_{R i}} F_{j i}\right),
$$

where $\tau_{R i}$ is the characteristic response time of the microrelief on this i impact ; $F_{j i}=$ $F_{j i}(v, \tau)$ is the function that is determined by the solution of the equation (8) at known frequency functions and.

Equation (9) indicates the method of accounting for the influence of the medium of usage on the microrelief of the body. When frequency functions are defined experimentally, formula (9) enables one to calculate the distribution before the exposure from the data on the distribution after the exposure. With unknown frequency functions, it is possible to determine them by comparing the experimental data on the functions before and after exposure, followed by prediction of the behavior of the body with prolonged use $[4,16]$. It is possible that the frequency functions may be useful in solving practical problems.

\section{Characterization of the morphological memory}

The complete characteristic of the body surface is the set of the state parameters of all the macromolecules that make up its microrelief. These state parameters are included in the distribution functions $\left\{\phi_{j}(\vec{X}, \tau)\right\}$, each of which can be represented as

$$
\phi_{j}(\vec{X}, \tau)=\phi_{j}(v, \tau) f_{j}(\vec{P}, \tau)_{v},
$$

where $f_{j}(\vec{P}, \tau)_{v}$ is the distribution density of the particle macromolecules in the state $(j, v)$ on the properties $\vec{P}$.

Given this, it is advisable to carry out a characterization of microrelief by a consistent definition of functions $\phi_{j}(v, \tau)$ and $f_{j}(\vec{P}, \tau)_{v}$ by measuring the volume of all the particles of microrelief, followed by the study of the bodies that have similar volume. And if the determination of the particle volumes is considered as the beginning of the study of the microrelief morphology, the value

$$
\phi(v, \tau)=\sum_{j} \phi_{j}(v, \tau)
$$

can be taken as the basic morphological characteristics of the microrelief.

According to equations (8) and (9), using experimental data on the distributions $\varphi_{j}(v, t)$ at the known frequency functions $\omega_{j}(\vec{y})$ and $\nu_{j}(\vec{y})$, the distributions $\phi_{j}\left(v, t_{F}\right)$ can be calculated. According to the equations (1)-(7), in the known frequency functions $\Omega_{j v}(\vec{y})$ and $\beta_{j v}(\vec{y})$ one can go from $\phi_{j}\left(v, t_{F}\right)$ to the distributions $\phi_{j}(v, t)$ at any time $t<t_{F}$. The given frequency function can be determined from the special experiments by comparison of the experimental data with functions $\phi_{j}(v, t)$, measured at different moments of time. After these experiments, it is possible for a computer to use the experimental data on the morphology of the body's surface to calculate the change in the distribution of its macromolecules 
on states in the formation process and new uses will arise. The possibility of creating such methods can be regarded as a manifestation of morphological memory of the body consisting in the long-term preservation of its nonequilibrium macromolecular structures, with the opportunity to study these structures without having to destroy the body. The formation of such structures can be considered as an accumulation of body information about the processes that lead to these structures' formation. The experimental determination of the functions $\phi_{j}(v, \tau)$ can be considered as an extraction of information from the morphological memory with data capacity $\varphi(v, \tau)$.

\section{Approaches to the use of morphological memory}

Apparently, morphological memory can be used to study the fast polymerization, the effects of short intense influences on the polymeric body and their slow degradation, i.e. where it is difficult to measure the speed of processes in situ. However, to realize this possibility, it is necessary to experimentally determine the frequency functions, participating in the equations (1), (7) and (8). Therefore, it is necessary to study the elementary processes that determine the function $\phi(v, \tau)$ on model bodies and to make sure that they leave a significant imprint on the microrelief. The obtained data on the frequency functions, supplemented by the boundary conditions of the solution of these equations, will lead to the formulation of a number of the boundary value problems on the morphological memory of the model bodies. Solution of these boundary value problems over a wide range of $\vec{y}$ will allow one to identify the main relations of the microrelief with the speeds of the elementary processes as well as to describe the response of the function $\phi(v, \tau)$ to the change of $\vec{y}$ and to develop algorithms for calculating the parameters of elementary processes from the experimental data about the function $\phi(v, \tau)$ at different $\vec{y}$. Currently, methods for solving such problems and the models of elementary processes are developed, and experimental data, allowing one to judge the main features of the microrelief and to make a phenomenological model of its formation, is accumulated [1-20]. For the quantitative study of the relation between the parameters of microrelief and the elementary processes, it is advisable to start with the simplest system, one in which the initiator molecules are rapidly converted into the nuclei of chain macromolecules, their number in the system reaching the value $N_{1}=C_{B} V_{0}$. The nuclei become larger, merging individual monomer molecules, while the elimination of the molecules joined by chains is unlikely $\left(\alpha_{1 v} C_{A} \gg \beta_{1 v}\right)$ and the merging frequency is independent of the size of the macromolecule $\left[\alpha_{1 v} \neq \alpha_{1 v}(v)\right]$, due to the constant number of merge sites. At the same time, $\Omega_{1 v} \gg \Omega_{2 v}, \Omega_{3 v}$, so that the aggregation during the time of the complete solvent removal $t_{F}$ does not occur, and equation $(7)$ is applicable, its solution at the boundary conditions $\phi(v, t)=0$ and $\left(G_{1 v} \phi_{1 v}-D_{1 v} \frac{\partial \phi_{1 v}}{\partial v}\right)_{v \rightarrow v_{n 1}}=N_{1} \delta(t)$ has the form:

$$
\phi_{1}(v, \tau)=N_{1}\left[(\pi A p)^{-1 / 2} \exp \left(-X_{-}^{2}\right)-\frac{1}{2 p} \exp \left(\frac{v-v_{n 1}}{p}\right) \operatorname{erfc}\left(X_{+}\right)\right] .
$$

Here, $\delta(t)$ is the Dirac delta function; $A=\int_{0}^{t} G_{1 v} d t ; p=D_{1 v} / G_{1 v} ; X_{ \pm}=\frac{v-v_{n 1} \pm A}{(4 \pi A)^{1 / 2}} ; v_{n 1}$ is the volume of the macromolecule nucleus; $\operatorname{erfc}\left(X_{+}\right)=\frac{2}{\sqrt{\pi}} \int_{X_{+}}^{\infty} e^{-X^{2}} d X$.

Distribution (12) corresponds to the following molecular weight distribution of the macromolecules in a given system

$$
\Psi_{1}(m, t) \equiv \frac{1}{N_{1}} \frac{\partial N_{1 m}}{\partial m}=B_{1} \exp \left[-\gamma\left(M-A_{1}\right)^{2}\right]-B_{2} \exp \left(M v_{0} / p\right)
$$


where $m=\left(m_{0} / v_{0}\right) v$ is the mass of the macromolecule with the volume $v$ if the mass of one link of the chain is $m_{0} ; N_{1 m}$ is the number of macromolecules with the mass less than $\mathrm{m} ; M=\left(m-m_{n}\right) / m_{0} ; m_{n}$ is the mass of the nucleus of the macromolecule; $A_{1}=A / v_{0}$; $B_{1}=\frac{v_{0}}{m_{0}} /(\pi A p)^{1 / 2} ; B_{2}=\frac{v_{0}}{2 m_{0} p} \operatorname{erf}\left[\gamma^{1 / 2}(M+A)\right] ; \gamma=v_{0} /\left(4 \pi A_{1}\right)$.

Equations (12) and (13) were obtained according to the kinetic models which are used in polymerization description [10,12,18-20], but using the function $\varphi_{1}(v, t)$. Similar relationships can be obtained to describe the aggregates, therefore one should detail the functions $\Omega_{j v}$ and $\beta_{j V}$ in accordance with the state parameters $\vec{X}$ of these particles. The sequence of work on creation and use of the morphological memory is shown in Fig. 1.

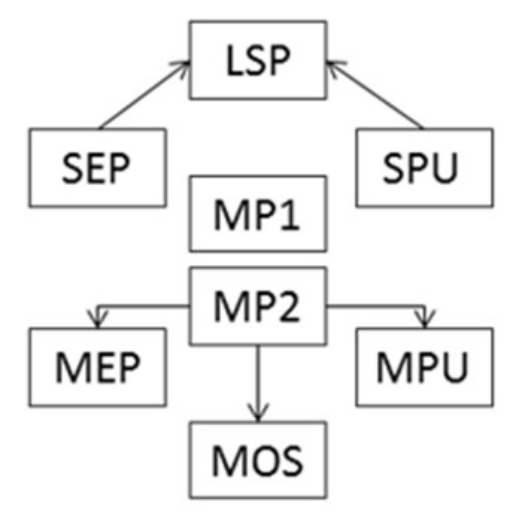

FIG. 1. Scheme of the work associated with the morphological memory. The complexes to resolve the following problems are shown. SEP - the study of elementary processes. LSP - laboratory synthesis of the product. SPU study of the changes in the product properties when it is used. MM1 - the accumulation of the information about the regularities of the product obtaining and use in the morphological memory. MM2 - development of models of product creation. MEP - models of the elementary processes. MOS - the model of optimal production synthesis. MPU - the model of the product behavior when it is used

\section{Morphological memory of the polystyrene bodies}

In Fig. 2, an electron microscopic image of the microrelief one of the polystyrene granules purchased from "Aldrich" is shown. Macromolecules of these granules were once formed in the devices of the company, and then became larger and aggregated in the body, which remained for a long time in the environment of use, and was then transferred to a microscope, where the surface of the body has taken the shape shown in Fig. 2. The same figure shows the image of the film obtained by dissolving these granules in $o$-xylene, followed by solvent evaporation, resulting in the formation of a film $[1,2]$. Other films and granules, randomly taken from commercial products, had a similar appearance.

All the examined bodies had the hierarchical microrelief available for the morphological characterization, wherein this microrelief was similar to that presented in the publications on polystyrene (e.g., in [21-23]) and other polymers (e.g., [24-26]).

The primary subnanoparticles of the size of $1-2 \mathrm{~nm}(\mathrm{j}=1)$, integrated in the chains and ring-shaped clusters, nanoparticles in the form of aggregates of these clusters $(j=2)$ and microparticles composed of these aggregates $(j=3)$ were the structural elements of the microrelief. 

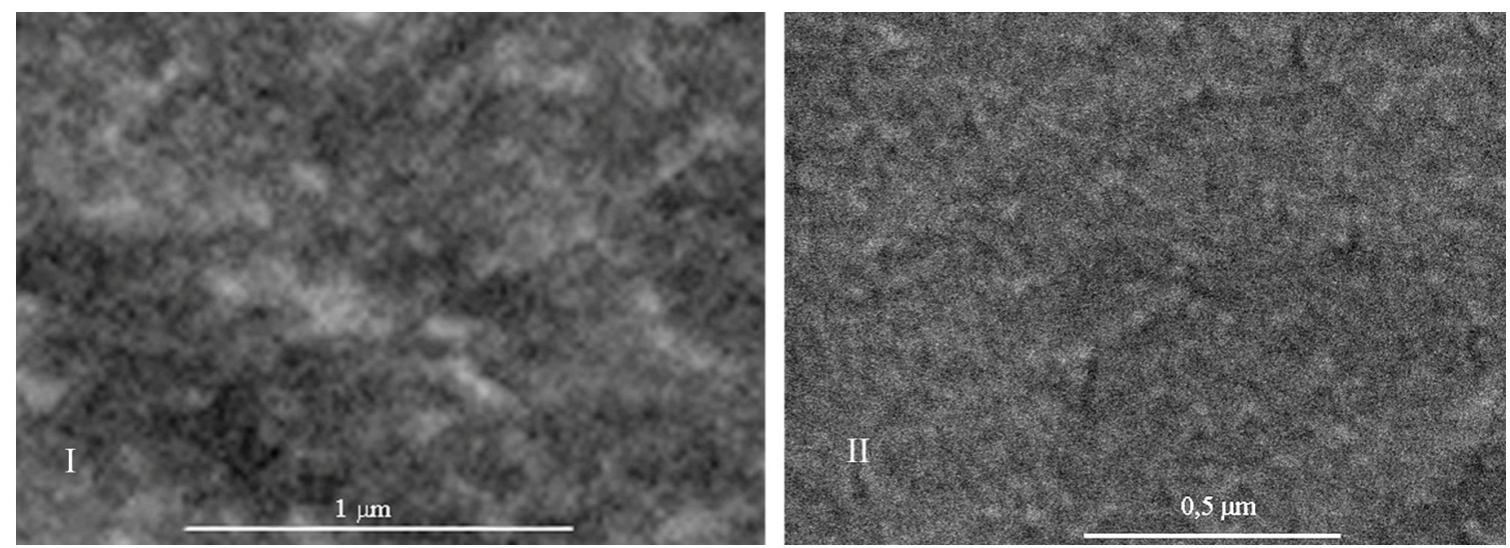

FIG. 2. The electron microscopic images of one of the areas of the surface of the granule and the film obtained by the method [1]. SamScan microscope (England) with a standard preparation of the samples and coating of the $\mathrm{Au}$ layer with the thickness of $5 \AA$. I - granule; II -film

TABLE 1. Limit volumes of the particles

\begin{tabular}{|c|c|c|c|}
\hline Volume, $\mathrm{nm}^{3}$ & Film & Granule & Granule after the contact \\
\hline$v_{10}$ & $2,2 \pm 0,3$ & $470 \pm 40$ & $19,7 \pm 4,2$ \\
\hline$v_{1 M} \cdot 10^{4}$ & $0,75 \pm 0,2$ & $3,4 \pm 0,4$ & $6,4 \pm 0,7$ \\
\hline$v_{20} \cdot 10^{5}$ & $0,09 \pm 0,02$ & $2,4 \pm 0,3$ & $3,6 \pm 0,5$ \\
\hline$v_{2 M} \cdot 10^{6}$ & $1,3 \pm 0,1$ & $13,1 \pm 0,4$ & $36,6 \pm 0,4$ \\
\hline
\end{tabular}

These particles were sufficiently ordered, allowing us to delineate the image of each of them to measure the area $\mathrm{S}$ and to find the parameter $v=S^{3 / 2}$ assigned as the volume of the particle. The average $\mathrm{j}^{\text {th }}$ type particle sizes within the composition of different $(\mathrm{j}+1)^{\text {th }}$ type particles did not differ remarkably, so that all the particles of each type were characterized by a single function:

$$
\theta_{j}(v)=\frac{1}{N_{j}} \int_{v_{0 j}}^{v} \phi_{j}(v, \tau) d v=B_{j v} / B_{j 0},
$$

where $B_{j v}$ is the number of measured particles of $\mathrm{j}^{\text {th }}$ type with the volume less than $v ; B_{j 0}$ is the total number of measured particles of $\mathrm{j}^{\text {th }}$ type; $v_{j 0}$ is the volume of the smallest of the detected particles.

Several functions $\theta_{j}(v)$ are shown in Figs. 3 and 4, where it is seen that the size of each particle type remained within a certain range of volumes $v_{j 0}<v<v_{M j}$, in which the value $\theta_{j}(v)$ increased from 0 to 1 (Table 1 ). The measurements showed that the interval $\Delta v_{j}=v_{M j}-v_{0 j}$ depended on the formation conditions and usage of the body. As it turned out, the interval $\Delta v_{j}$ for the nanoparticles of the film obtained by evaporation of a fullerene $\left(\mathrm{C}_{60}\right)$-containing solution, was dependent on the concentration of fullerene according to the relationship:

$$
\Delta v_{2}=\Delta v_{20}\left(1+\chi_{F} M_{F} / M_{M}\right) \text {, }
$$

where $\Delta v_{20}$ is an interval without fullerene; $M_{F}$ and $M_{M}$ are masses of fullerene and polystyrene introduced in the original solution at $M_{F} / M_{M}=\left(3 \cdot 10^{-4} \div 1 \cdot 10^{-3}\right) ; \chi_{F}=$ $(1552 \pm 42)$ is an empirical coefficient. 


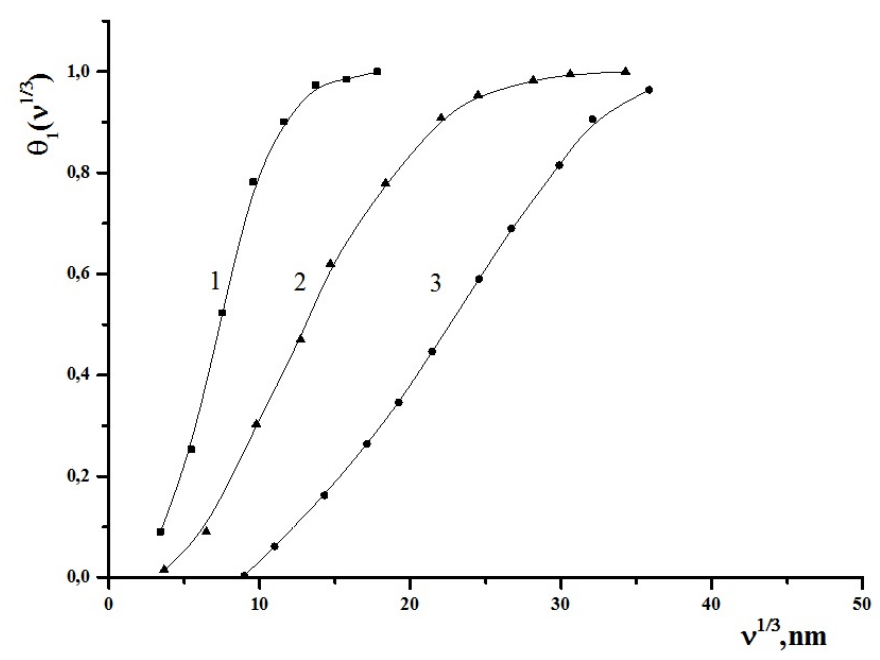

FIG. 3. Distribution functions $\theta_{1}(v)$ of subnanoparticles of the film and granule microrelief on the volume before and after a short introduction in toluene. 1 - film, 2 - granule before to the contact with toluene, 3 - granule after the contact with toluene

TABLE 2. Limit volumes of the nanoparticles of the film with different content of the fullerene

\begin{tabular}{|c|c|c|}
\hline $\mathrm{M}_{F} / \mathrm{M}_{M}$ & $v_{0 j} \cdot 10^{5} \mathrm{~nm}^{3}$ & $v_{M j} \cdot 10^{6} \mathrm{~nm}^{3}$ \\
\hline 0 & 0,09 & 1,3 \\
\hline $3 \cdot 10^{-4}$ & 0,15 & 1,5 \\
\hline $10^{-3}$ & 0,22 & 1,7 \\
\hline
\end{tabular}

The concentration of fullerene also affected the maximum volume of the nanoparticles:

$$
v_{M 2}=v_{M 0}\left[\left(1+\chi_{v}\left(M_{F} / M_{M}\right)^{k}\right]\right.
$$

where $v_{M 0}$ is the maximum volume at $M_{F}=0 ; \chi_{v}=20,5 \pm 0,1$ and $\mathrm{k}=0.61 \pm 0.02$ are empirical coefficients (Table 2).

Equations (15) and (16) indicate that the microrelief of the film memorized the quantity of the fullerene in the solution in which the film was formed, whereas the granule subjected to the partial dissolution remembered what happened to it at dissolution. This is indicated by the data in Figs. 3 and 4, which show that the granule placement into the stream of toluene at a temperature of $300{ }^{\circ} \mathrm{K}$ for $\tau=60 \mathrm{~s}$ resulted in a significant change of the distribution functions $\theta_{j}(v)$. This change can be characterized by the parameter $P_{j}$ :

$$
\theta_{j}(v)_{t_{F}}=\theta_{j}\left(v+P_{j}\right)_{\tau},
$$

where $\theta_{j}(v)_{t_{F}}$ and $\theta_{j}\left(v+P_{j}\right)_{\tau}$ are the functions $\theta_{j}(v)$ before and after the contact with toluene when the volume of the particles was equal to $v$ and $v+P_{j}$, respectively.

According to the data in Figs. 3-5, the parameter $P_{j}$ was equal to:

$$
P_{j}=v_{j \tau}-v=P_{0 j}\left(v / v_{0 j}\right)^{q_{j}}
$$

where $v_{j \tau}$ is the volume of the particle of $\mathrm{j}^{\text {th }}$ type, which volume equaled to $v$ before the contact at the moment $\tau ; P_{0 j}=v_{j \tau}-v_{0 j}$ and $q_{j}$ are empirical parameters (Fig. 5). The 


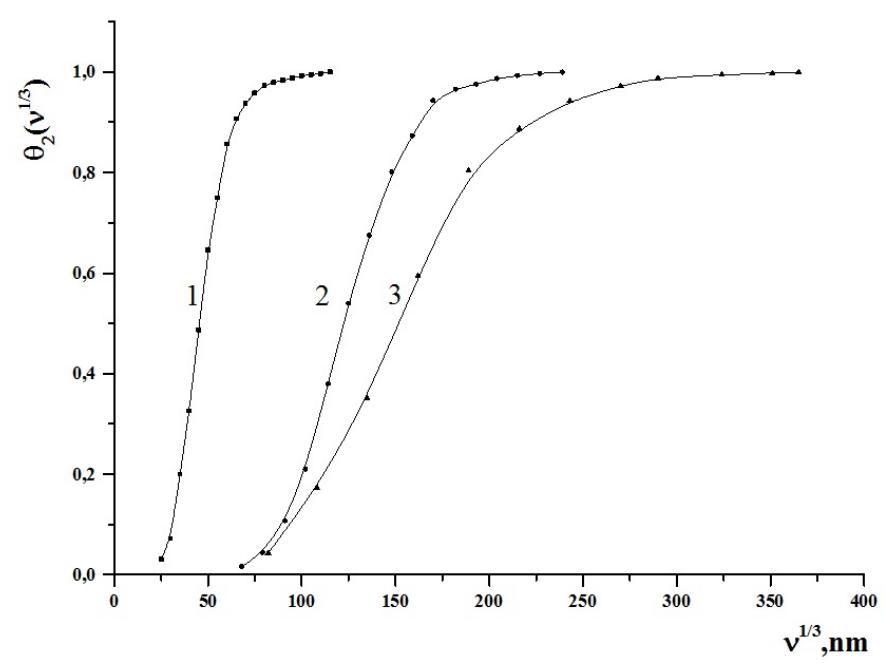

FIG. 4. Distribution functions $\theta_{2}(v)$ on volume of nanoparticles of the film and granule. 1 - film, 2 - granule before to the contact with toluene, 3 granule after the contact with toluene

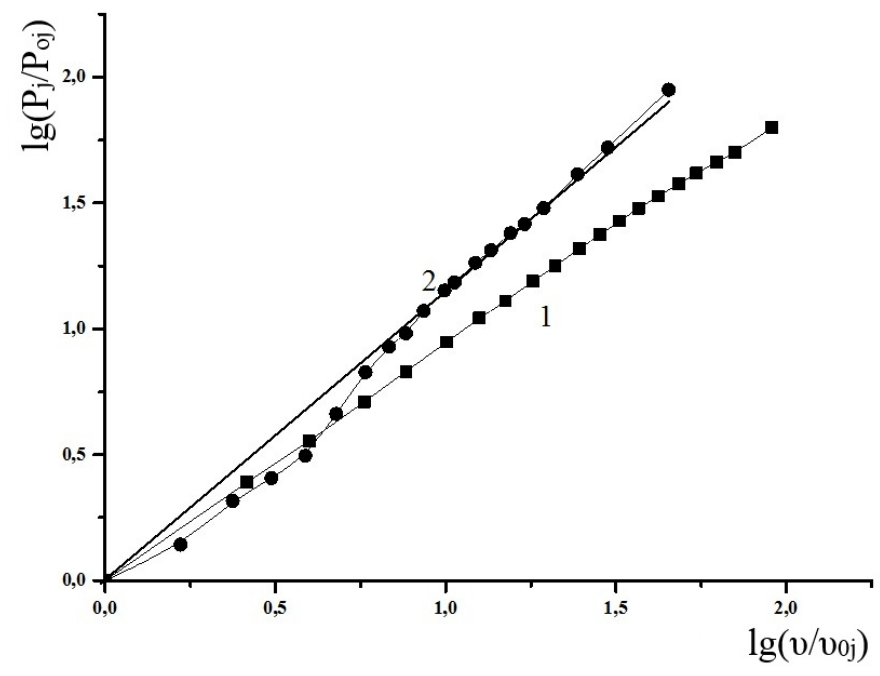

Fig. 5. Parameter $\mathrm{P}_{j}$ of the subnano- and nanoparticles of the granules microrelief. 1 - subnanoparticles at $\mathrm{P}_{01} \mathrm{q}=0.98 ; 2-$ nanoparticles at $\mathrm{P}_{02} \mathrm{q}=1,27$

volume $v_{j \tau}$ could increase due to the capture of toluene molecules by the particles and decrease due to the release of macromolecules. Therefore:

$$
P_{j}=\int_{0}^{\tau}\left(\omega_{j} a_{j}-\nu_{j} b_{j}\right) d \tau^{*},
$$

where $\omega_{j}$ and $\nu_{j}$ are the frequencies of events leading to the increase and decrease of $v_{j \tau} ; a_{j}$ and $b_{j}$ are the changes of $v_{j \tau}$ at a single event at $0<\tau^{*}<\tau$.

From equations (16) and (17), it follows that during contact with toluene and its subsequent removal from the granules, the following conditions were fulfilled:

$$
\begin{gathered}
\omega_{j}=\omega_{j}\left(\tau^{*}\right)\left(v / v_{0 j}\right)^{q_{j}}, \\
\nu_{j}=\nu_{j}\left(\tau^{*}\right) N_{j-1}^{q_{i}},
\end{gathered}
$$


where $N_{j-1}=v / \bar{a}_{j-1}$ is the number of the particles of $(\mathrm{j}-1)^{t h}$ type in the particle of $\mathrm{j}^{\text {th }}$ type with the volume $v ; \bar{a}_{j-1}$ is the average volume per $\mathrm{j}^{\text {th }}$ type particle in the volume $v ; \omega_{j}\left(\tau^{*}\right)$ and $\nu_{j}\left(\tau^{*}\right)$ are the frequency functions, characterizing the probability of the participation of a single particle of the $\mathrm{j}^{\text {th }}$ type in the change of $v$ per unit time.

States (18)-(21) can be considered as an indication of the fact that the capture of the toluene molecules by the granule causes their penetration in the volume of nanoand subnanoparticles with solvation of macromolecules, causing the weakening of the bond between the macromolecules and providing their removal from the subnanoparticles with a similar probability $\left(q_{1} \rightarrow 1\right)$.

Toluene molecules, which penetrated into the subnanoparticles during contact, were removed from the subnanoparticles after contact, so when in the microscope, their parameter $P_{j}$ is similar to:

$$
P_{1}=-N_{0}(v) \int_{0}^{\tau} \nu_{1}\left(\tau^{*}\right) b_{1} d \tau^{*},
$$

where $N_{0}(v)$ is the quantity of the macromolecules in the subnanoparticle of the volume $v$ before the contact.

The parameter $P_{j}$ of the nanoparticles in the microscope is equal to:

$$
P_{2}=\left(v / v_{0 j}\right)^{q_{2}} \int_{0}^{\tau} \omega_{2}\left(\tau^{*}\right) a_{2} d \tau^{*} .
$$

Their frequency function $\omega_{2}\left(\tau^{*}\right)$ increased at the contact due to the capture of toluene, and decreased after the contact due to toluene removal from the granules. Additionally, if the structure of nanoparticles changed reversibly during toluene capture, the condition $P_{2}=0$ would be fulfilled. However, solvation of the macromolecules lead to irreversible changes in the location of subnanoparticles in the volume of nanoparticles, so $P_{2}>0$.

Equations (18)-(23) make it possible to determine the frequency functions $\omega_{j}\left(\tau^{*}\right)$ and $\nu_{j}\left(\tau^{*}\right)$, information on which is stored in the function $P_{j}=P_{j}(v)$. One can try to simulate the frequency functions using equations (7)-(11).

Considering the above, it can be argued that the studied films and granules of polystyrene possessed the morphological memory. These granules maintained their nonequilibrium hierarchical structures, formed on their surface, for the long time in such a state, thus allowing an approach for the identification of kinetic equations for the hierarchies' formation.

\section{Conclusion}

Experiments with polystyrene showed the concept of the morphological memory of the polymeric bodies as the ability of their surface microrelief to maintain long-term nonequilibrium macromolecular structures that can be effectively used in the study of polymerization and in the search for the optimal conditions for polymer synthesis. It was found that the proposed models for the formation of polymers, devoid of arbitrary assumptions, could be the basis for creating a computer system for the application of morphological memory to determine the rates of elementary processes and to predict the behavior of polymers during their prolonged use. 


\section{Acknowledgment}

The work was supported by Russia Foundation for Basic Research (grant N 15-4303034-a).

\section{References}

[1] Alekseeva O.V., Bagrovskaya N.A., Kuzmin S.M., Noskov A.V., Melikhov I.V., Rudin V.N. Influence of fullerene additives on the structure of the polystyrene films. Russ. J. Phys. Chem., 2009, 83(7), P. 1320-1326.

[2] Alekseeva O.V, Bagrovskaya N.A., Kuzmin S.M., Noskov A.V., Melikhov I.V., Rudin V.N. Effect of $\mathrm{C}_{60}$ Doping on the Structure and Properties of Polystyrene. In Handbook on Fullerene: Syntesis, Properties and Applcations ISBN 978-1-62100-429-5. Editor:R.F.Verner, C.Benvegnu, P. 439-456. 2012 Nova Science Publishers, Inc. New York, 558 p.

[3] Brinker C.J., Scherer G.W. Sol-Gel Science. The Physics and Chemistry of Sol-Gel Processing. San Diego, CA: Academic Press, 1990, 908 p.

[4] Salamone C. (Ed). Polymeric Material Encyclopedia. Boca Raton TL: CRC Press, 1996.

[5] Korolev G.V., Mogilevich M.M. Three-dimensional radical polymerization. Mesh and hyperbranched polymers. Khimia. St. Petersburg, 2006.

[6] Kabanov V.A. Selected Works. Nauka, Moscow, 2010, 603 p.

[7] Ferreiro, V., Douglas, J. F., Warren, J., and Karim, A. Non-Equilibrium Pattern Formation in the Crystallization of Polymer Blend Films. Physical Review E, 2002, 65(4), P. 042802:1-4.

[8] Irzhak V.I., Mezhikovsky S.M. Kinetics of oligomer curing. Russ. Chem. Rev., 2008, 77(1), P. 78-104.

[9] Emanuel N.M., Buchachenko A.L. Chemical physics of molecular degradation and stabilization of polymers. Moscow, Nauka, 1988, 368 p.

[10] Grosberg A.Yu., Khokhlov A.R. Polymers and biopolymers from the point of view of physics. Dolgoprudny: Publishing House "Intellect", 2010, 304 p.

[11] Palmer R.E., Pratonter S., Boyen H.-G. Nanostructured Surfaces from size-selected clusters. Nature Materials, 2003, 2(7), P. 443-448.

[12] Askadskii A.A., Khokhlov A.R. Introduction to physical chemistry of polymers. Moscow, Nauchny Mir, 2009, 384 p.

[13] Melihov I.V. An evolutionary approach to the creation of nanostructures. Nanosystems: physics, chemistry, mathematics, 2010, 1(1), P. 148-155.

[14] Melikhov I.V., Physico-chemical evolution of solid. Moscow, Binom, 2006, 309 p.

[15] Yampolsky Yu.P. Methods of studying the free volume in polymers. Russ. Chem. Rev., 2007, 76(1), P. $66-87$.

[16] Nalwes H.S. (Ed). Handbook of Nanostructured Materials and Nanotechnology, v 5. Organics, Polymer and Biological Compounds. Boston: Acad. Press, 2000.

[17] Tant M.R., Connell J.W., McManus H.L.N.(Eds). High Temperature Properties and Applications of Polymeric Materials. ACS Symposium Series. V 603. American Chemical Society, Washington Dc, 1995.

[18] Mozhikovsky S.M., Arinshtein A.E., Deberdeev R.Ya. Oligomeric state of matter. Moscow, Nauka, 2005, $252 \mathrm{p}$.

[19] Volfson S.A., Enikolopyan I.S. The calculation of high-performance polymerization processes. Moscow, Khimia, 1980, $312 \mathrm{p}$.

[20] Fredrickson G.H. The Equilibrium Theory of Inhomogeneous Polymers. Oxford: University Press, 2006.

[21] Simon P., Huhle R., Lehmann M., Lichte H., Monter D., Bieber T., Reschetilowski V., at all. Electron Holography on Beam Sensitive Materials: Organic Polymers and Mesoporous Silica. Chem.Mater., 2002, 14, P. 1505-1514.

[22] Vang D., Zhu J., Yao Q., Wilkie C.A. A Comparison of Various Methods for the Preparation of Polystyrene an Poly(methyl methacrylate) clay Nanocomposites. Chem.Mater., 2002, 14(9), P. 38373843.

[23] Dutschke A., Diegelmann C., Lobmann P. Nucleation and Growth of $\mathrm{TiO}_{2}$ Thin Films on Modified Polystyrene Surfaces. Chem.Mater., 2003, 15(18), P. 3501-3506.

[24] Seeman N.C. DNA in a material world. Nature, 2003, 421(6921), P. 427-431. 
[25] Egen M., Voss R., Griesebock B., Zentel R. Heterostructures of Polymer Photonic Crystal Films. Chem.Mater., 2003, 15(20), P. 3786-3790.

[26] Donald A.M. The use of environmental scanning electron microscopy for imaging wet and insulating materials. Nature Materials, 2003, 2(8), P. 511-516. 